\title{
@creative
}

\section{Involutes of polygons of constant width in Minkowski planes}

\author{
Marcos Craizer* \\ Departamento de Matemática- PUC-Rio, Rio de Janeiro, Brazil \\ Horst Martini \\ Faculty of Mathematics, University of Technology, 09107 Chemnitz, Germany
}

Received 2 July 2015, accepted 21 August 2015, published online 24 September 2015

\begin{abstract}
Consider a convex polygon $P$ in the plane, and denote by $U$ a homothetical copy of the vector sum of $P$ and $-P$. Then the polygon $U$, as unit ball, induces a norm such that, with respect to this norm, $P$ has constant Minkowskian width. We define notions like Minkowskian curvature, evolutes and involutes for polygons of constant $U$-width, and we prove that many properties of the smooth case, which is already completely studied, are preserved. The iteration of involutes generates a pair of sequences of polygons of constant width with respect to the Minkowski norm and its dual norm, respectively. We prove that these sequences are converging to symmetric polygons with the same center, which can be regarded as a central point of the polygon $P$.
\end{abstract}

Keywords: Area evolute, Barbier's theorem, center symmetry set, curvature, curves of constant width, discrete differential geometry, evolutes, Minkowski geometry, normed plane, equidistants, involutes, support function, width function.

Math. Subj. Class.: 52A10, 52A21, 53A15, 53A40

\section{Introduction}

A Minkowski or normed plane is a 2-dimensional vector space with a norm. This norm is induced by its unit ball $U$, which is a compact, convex set centered at the origin (or, shortly, centered). Thus, we write $\left(\mathbb{R}^{2}, U\right)$ for a Minkowski plane with unit ball $U$, whose boundary is the unit circle of $\left(\mathbb{R}^{2}, U\right)$. The geometry of normed planes and spaces, usually

\footnotetext{
${ }^{*}$ The first named author wants to thank CNPq for financial support during the preparation of this manuscript.

E-mail addresses: craizer@puc-rio.br (Marcos Craizer), martini@ mathematik.tu-chemnitz.de (Horst Martini)
} 
called Minkowski Geometry (see [21], [14], and [13]), is strongly related to and influenced by the fields of Convexity, Banach Space Theory, Finsler Geometry and, more recently, Discrete and Computational Geometry. The present paper can be considered as one of the possibly first contributions to Discrete Differential Geometry in the spirit of Minkowski Geometry. The study of special types of curves in Minkowski planes is a promising subject (see the survey [15]), and the particular case of curves of constant Minkowskian width has been studied for a long time (see [3], [4], [11], and $\S 2$ of [13]). A curve $\gamma$ has constant Minkowskian width with respect to the unit ball $U$ or, shortly, constant $U$-width, if $h(\gamma)+$ $h(-\gamma)$ is constant with respect to the norm induced by $U$, where $h(\gamma)$ denotes the support function of $\gamma$. Another concept from the classical theory of planar curves important for our paper is that of involutes and evolutes; see, e.g., Chapter 5 of [8] and, respectively, [9]. For natural generalizations of involutes, which also might be extended from the Euclidean case to normed planes, we refer to [18] and [2]. And in [20] it is shown how the concept of evolutes and involutes can help to construct curves of constant width in the Euclidean plane.

In this paper, we consider convex polygons $P$ of constant Minkowskian width in a normed plane, for short calling them $C W$-polygons. If $P$ is a $\mathrm{CW}$ polygon, then the unit ball $U$ is necessarily a centered polygon whose sides and diagonals are suitably parallel to corresponding sides and diagonals of $P$ (sometimes with diagonals suitably meaning also sides; see $\S \S 2.1$ below). If, in particular, $U$ is homothetic to $P+(-P)$, then, and only then, $P$ is of constant $U$-width in the Minkowski plane induced by $U$.

There are many results concerning smooth $C W$ curves in normed planes: Barbier's theorem fixing their circumference only by the diameter of the curve (cf. [16] and [12]); relations between curvature, evolutes, involutes, and equidistants (see [19] and, for applications of Minkowskian evolutes in computer graphics, [1]); mixed areas, and the relation between the area and length of a CW curve cut off along a diameter (see [3], (2.1)). In this paper we prove corresponding results for $C W$ polygons. We note that our results are direct discretizations of the corresponding results for the smooth case, where the derivatives and integrals are replaced by differences and sums. It is meant in this sense that the results of this paper can be considered as one of the first contributions to Discrete Differential Geometry in the framework of normed planes.

Among the $U$-equidistants of a smooth $\mathrm{CW}$ curve $\gamma$, there is a particular one called central equidistant. The central equidistant of $\gamma$ coincides with its area evolute, while the evolute of $\gamma$ coincides with its center symmetry set (see [6] and [7]). We show that for a $\mathrm{CW}$ polygon $P$ the same results hold: The central equidistant $M$ coincides with the area evolute, and the evolute $E$ coincides with the central symmetry set (see [5]). Since the equidistants of $P$ are the involutes of $E$, we shall choose the central equidistant as a representative of them, and we write $M=\operatorname{Inv}(E)$.

For a Minkowski plane whose unit ball $U$ is a centered convex (2n)-gon, the dual unit ball $V$ is also a centered convex (2n)-gon with diagonals parallel to the sides of $U$, and the sides parallel to diagonals of $U$. As in the smooth case (cf. [6]), the involutes of the central equidistant of $P$ form a one-parameter family of polygons having constant $V$-width. This one-parameter family consists of the $V$-equidistants of any of its members, and we shall choose the central equidistant $N$ as its representative. Thus we write $N=\operatorname{Inv}(M)$. In [6] it is proved that, for smooth curves, the analogous involute $N$ is contained in the region bounded by $M$ and has smaller or equal signed area. In this paper we prove the corresponding fact for polygons, namely, that $N$ is contained in the region bounded by $M$ 
and the signed area of $N$ is not larger than the signed area of $M$.

What happens if we iterate the involutes? Let $N(0)=E, M(0)=M, N(1)=N$ and define $M(k)=\operatorname{Inv}(N(k)), N(k+1)=\operatorname{Inv}(M(k))$. Then we obtain two sequences $M(k)$ and $N(k)$, the first being of constant $U$-width and the second of constant $V$-width. Moreover, we have

$$
\overline{N(0)} \supset \overline{M(0)} \supset \overline{N(1)} \supset \overline{M(1)} \supset \ldots,
$$

where $\bar{R}$ denotes the closure of the region bounded by $R$. Denoting by $O=O(P)$ the intersection of all these sets, we shall prove that $O$ is in fact a single point. Another form of describing the convergence of $M(k)$ and $N(k)$ to $O$ is as follows: For fixed $c$ and $d$, consider the sequences $M(k)+c U$ of polygons of constant $U$-width, and the sequences $N(k)+d V$ of polygons of constant $V$-width. Then these sequences are converging to $O+c U$ and $O+d V$, respectively, which are $U$ - and $V$-balls centered at $O$. For smooth curves the analogous results were proved in [6].

Our paper is organized as follows: In Section 2 we describe geometrically the unit ball of a Minkowski plane for which a given convex polygon has constant Minkowskian width. In Section 3, we define Minkowskian curvature, evolutes and involutes for $\mathrm{CW}$ polygons and prove many properties of them. In Section 4 we consider the involute of the central equidistant, and in Section 5 we prove that the involutes iterates are converging to a single point.

\section{Polygonal Minkowskian balls, their duals, and constant Minkowski- an width}

Since faces and also width functions of convex sets behave additively under (vector or) Minkowski addition, it is clear that a polygon $P$ is of constant Minkowskian width if and only if $P+(-P)$ is a homothetical copy of the unit ball $U$ of the respective normed plane; see, e.g., $\S \S 2.3$ of [13]. If, moreover, the homothety of $U$ and $P+(-P)$ is only possible when $P$ itself is already centrally symmetric, then the only sets of constant $U$-width are the balls of that norm; cf., e.g., [22]. In the following we will have a closer look at various geometric relations between polygons $P$ of constant $U$-width and the unit ball $U$, since we need them later.

Thus, let $P$ be an arbitrary planar convex polygon. By an abuse of notation, we shall denote by the same letter $P$ also the set of vertices of the polygon, the closed polygonal arc formed by the union of its sides, and the convex region bounded by $P$.

\subsection{A centered polygon with parallel sides and diagonals}

Assume that $P=\left\{P_{1}, \ldots, P_{2 n}\right\}$ is a planar convex polygon with parallel opposite sides, i.e., the segments $P_{i} P_{i+1}$ and $P_{i+n} P_{i+n+1}, 1 \leq i \leq n$, are parallel.

Lemma 2.1. Fix an origin $Z$ and take $U_{1}$ such that $U_{1}-Z=\frac{1}{2 a}\left(P_{1}-P_{1+n}\right)$, for some $a>0$. Consider the polygon $U$ whose vertices are

$$
U_{i}=Z+\frac{1}{2 a}\left(P_{i}-P_{i+n}\right)
$$

$1 \leq i \leq 2 n$. Then $U$ is convex, symmetric with respect to $Z, U_{i+1}-U_{i} \| P_{i+1}-P_{i}$ and $U_{i}-Z \| P_{i}-P_{i+n}$ for $1 \leq i \leq n$ (see Figure 1 ). Moreover, $U$ is the unique polygon with these properties. 


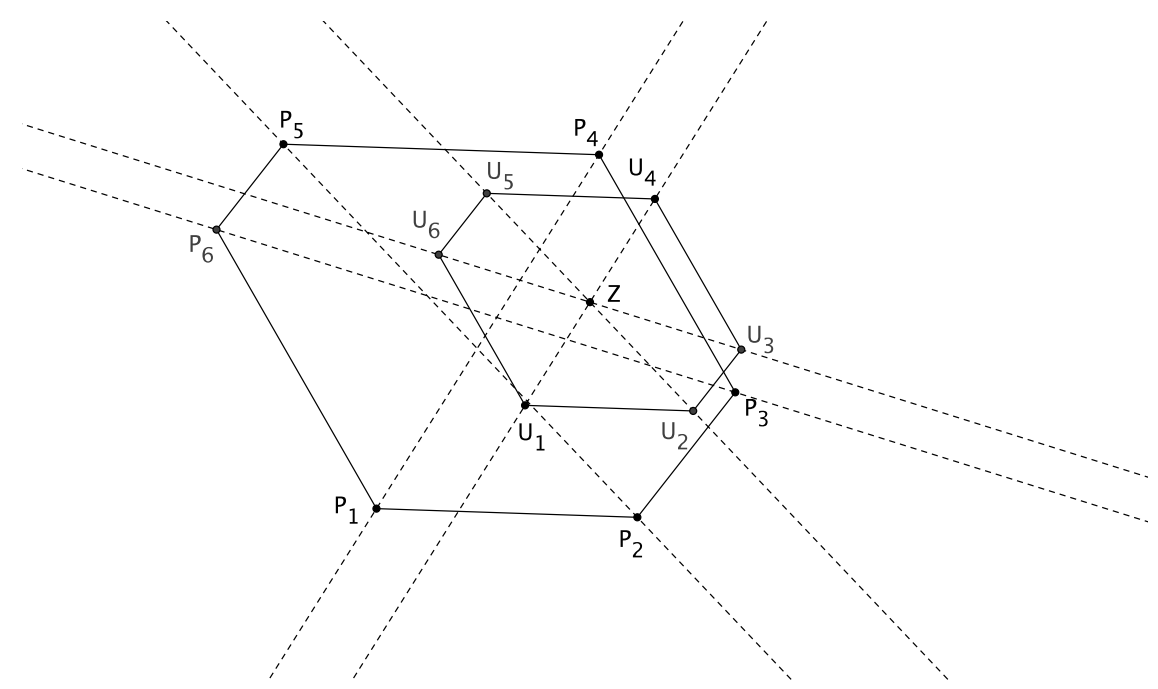

Figure 1: A hexagon $P$ with parallel opposite sides and the corresponding homothet $U$ of $P+(-P)$.

Proof. It is clear that $U$ is symmetric with respect to $Z, U_{i+1}-U_{i} \| P_{i+1}-P_{i}$ and $U_{i}-Z \| P_{i}-P_{i+n}$ for $1 \leq i \leq n$. Moreover $U_{i+1}-U_{i}$ has the same orientation as $P_{i+1}-P_{i}$, which implies that $U$ is convex.

To prove the uniqueness of $U$, observe that the point $U_{2}$ is obtained as the intersection of the lines parallel to $P_{1} P_{2}$ through $U_{1}$ and parallel to $P_{2} P_{2+n}$ through $Z$. The points $U_{3}, \ldots, U_{n}$ are obtained inductively in a similar way, while $U_{n+1}, . ., U_{2 n}$ are reflections of $U_{1}, \ldots U_{n}$ with respect to $Z$.

Consider now a convex polygon $\tilde{P}=\left\{\tilde{P}_{1}, \ldots, \tilde{P}_{k}\right\}$ that has not necessarily all opposite sides parallel. Suppose that exactly $0 \leq j \leq \frac{k}{2}$ pairs are parallel. Our next lemma shows that the list of vertices of this polygon can be re-written as $P=\left\{P_{1}, P_{2}, . ., P_{2 n}\right\}, n=k-j$, with "parallel opposite sides" in a broader sense.

Lemma 2.2. We may re-write the list of vertices of $\tilde{P}$ as $\left\{P_{1}, P_{2}, . ., P_{2 n}\right\}$ such that, for each $1 \leq i \leq n, P_{i} P_{i+1}$ is parallel to $P_{i+n} P_{i+n+1}$ or else one of these sides, say $P_{i+n} P_{i+n+1}$, degenerates to a point, in which case the other side $P_{i} P_{i+1}$ is not degenerated and the line through $P_{i+n}=P_{i+n+1}$ parallel to $P_{i} P_{i+1}$ is outside $P$ (see Figure 2).

Proof. The polygon $\tilde{P}=\left\{\tilde{P}_{1}, \ldots, \tilde{P}_{k}\right\}$ defines exactly $n=k-j$ directions $\theta_{1}, \ldots, \theta_{n}$, in increasing order, in the plane. We may assume that $\tilde{P}_{1} \tilde{P}_{2}$ is in direction $\theta_{1}$ and define $P_{1}=\tilde{P}_{1}, P_{2}=\tilde{P}_{2}$. For the induction step write $P_{i}=\tilde{P}_{l}$. If $P_{i} \tilde{P}_{l+1}$ is in direction $\theta_{i}$, define $P_{i+1}=\tilde{P}_{l+1}$, otherwise define $P_{i+1}=\tilde{P}_{l}$. It is now easy to verify that the polygon $P=\left\{P_{1}, P_{2}, . ., P_{2 n}\right\}$ satisfies the properties of the lemma.

The construction of Lemma 2.1 can be applied to the polygon $P$ obtained in Lemma 2.2 (see Figure 2). If, for example, $P$ is a triangle, then $P+(-P)$ is an affinely regular hexagon (see Figure 3). From now on, we shall assume that $Z$ coincides with the origin of $\mathbb{R}^{2}$ and that $P=\left\{P_{1}, \ldots, P_{2 n}\right\}$, with $P_{i} P_{i+1}$ parallel to $U_{i} U_{i+1}$. 


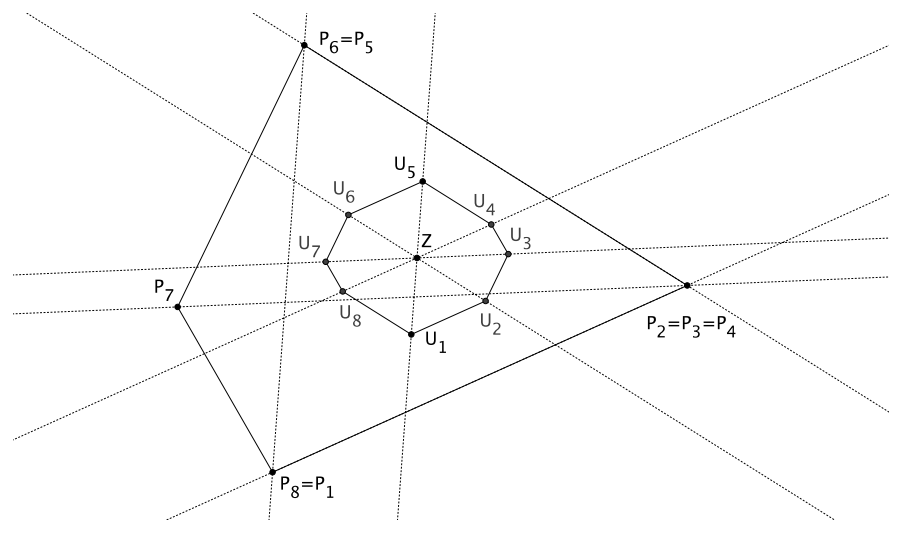

Figure 2: A quadrangle and the corresponding symmetric octagon.

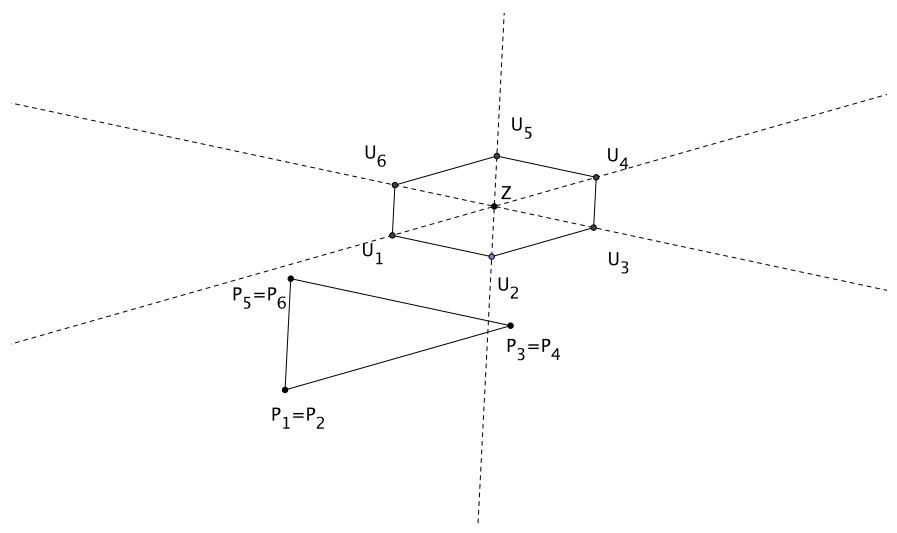

Figure 3: When $P$ is a triangle of constant $U$-width, then $U$ is an affinely regular hexagon.

\subsection{The dual Minkowskian ball}

Now we introduce the type of duality which is very useful for our investigations. Let $\left(\mathbb{R}^{2}\right)^{*}$ denote the space of linear functionals in $\mathbb{R}^{2}$. The dual norm in $\left(\mathbb{R}^{2}\right)^{*}$ is defined as

$$
\|f\|=\sup \{f(u), u \in U\} .
$$

We shall identify $\left(\mathbb{R}^{2}\right)^{*}$ with $\mathbb{R}^{2}$ by $f(\cdot)=[\cdot, v]$, where $[\cdot, \cdot]$ denotes the determinant of a pair of planar vectors. Under this identification, the dual norm in $\mathbb{R}^{2}$ is given by

$$
\|v\|=\sup \{[u, v], u \in U\} .
$$

We shall construct below a centered polygon $V$ such that, for $v$ in any side of $V$, we have $\|v\|=1$. Such a polygon defines a Minkowski norm equivalent to the dual norm of $U$.

Now assume that the unit ball $U$ is a centered polygon with vertices $\left\{U_{1}, \ldots, U_{2 n}\right\}$, $U_{i+n}=-U_{i}, 1 \leq i \leq n$. Define the polygon $V$ with vertices

$$
V_{i+\frac{1}{2}}=\frac{U_{i+1}-U_{i}}{\left[U_{i}, U_{i+1}\right]}
$$


Observe that $V_{i+n+\frac{1}{2}}=-V_{i+\frac{1}{2}}$, i.e., $V$ is centered. Now $\left[V_{i+\frac{1}{2}}-V_{i-\frac{1}{2}}, U_{i}\right]=0$, which implies that $V_{i+\frac{1}{2}}-V_{i-\frac{1}{2}}=-a U_{i}$. Multiplying both sides by $V_{i+\frac{1}{2}}$ we obtain

$$
U_{i}=-\frac{V_{i+\frac{1}{2}}-V_{i-\frac{1}{2}}}{\left[V_{i-\frac{1}{2}}, V_{i+\frac{1}{2}}\right]},
$$

for $1 \leq i \leq 2 n$.

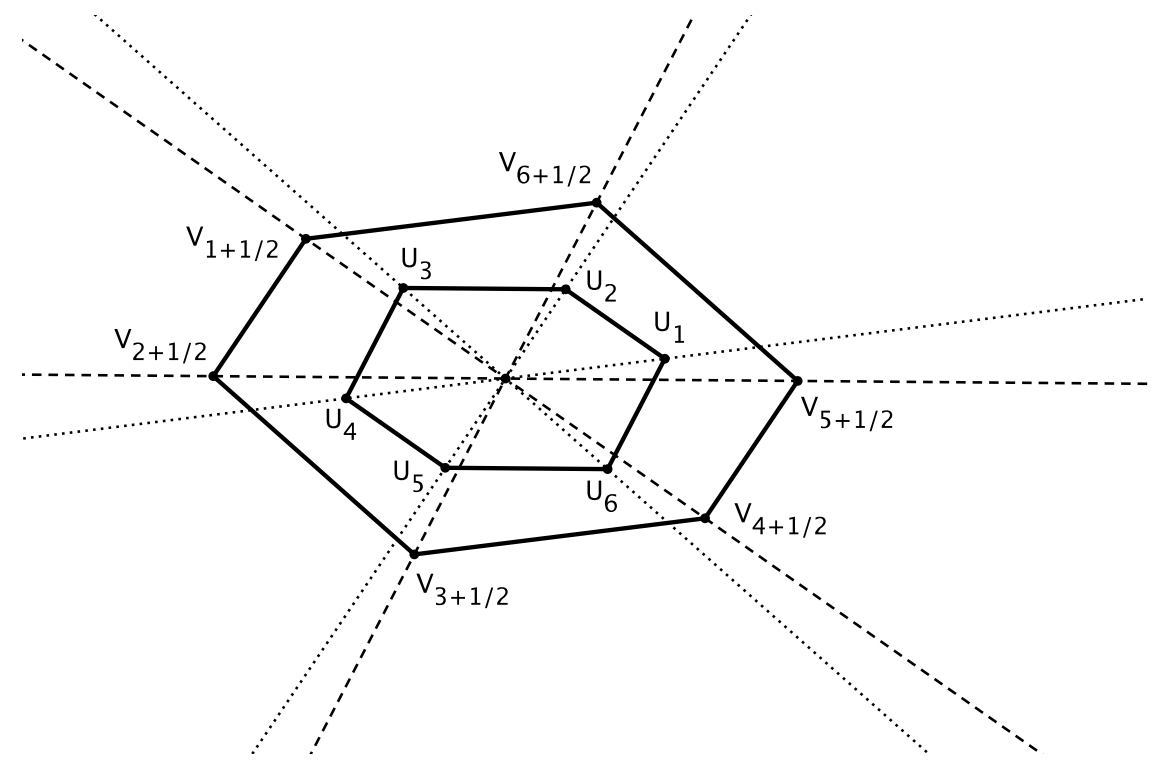

Figure 4: The centered hexagon $U$ and its dual $V$.

Lemma 2.3. The polygon $V$ is the dual unit ball.

Proof. We have that, for $1 \leq i \leq 2 n$,

$$
\left[t U_{i}+(1-t) U_{i+1}, V_{i+\frac{1}{2}}\right]=1
$$

for any $t \in \mathbb{R}$ and for $j \notin\{i, i+1\},\left[U_{j}, V_{i+\frac{1}{2}}\right] \leq 1$. This implies that the vertex $V_{i+\frac{1}{2}}$ is from the dual unit circle. Moreover,

$$
\left[U_{i}, t V_{i-\frac{1}{2}}+(1-t) V_{i+\frac{1}{2}}\right]=1
$$

and for $j \neq i$ we have $\left[U_{j}, t V_{i-\frac{1}{2}}+(1-t) V_{i+\frac{1}{2}}\right] \leq 1$, which implies that also the side $t V_{i-\frac{1}{2}}+(1-t) V_{i+\frac{1}{2}}$ is from the dual unit circle.

\subsection{Polygons of constant Minkowskian width}

Consider a Minkowski plane $\left(\mathbb{R}^{2}, U\right)$, and let $P$ be a convex curve. For $f$ in the dual unit ball, the support function $h(P)(f)$ of $P$ at $f$ is defined as

$$
h(P)(f)=\sup \{f(p), p \in P\} .
$$


The width of $P$ in the direction $f$ is defined as $w(P)(f)=h(P)(f)+h(P)(-f)$. We say that $P$ is of constant Minkowskian width if $w(P)(f)$ does not depend on $f$.

Consider now a Minkowski plane whose unit ball $U$ is a centered polygon, and let $P$ be a polygon with parallel corresponding sides and diagonals.

Lemma 2.4. In the Minkowski plane $\left(\mathbb{R}^{2}, U\right)$, P has constant $U$-width.

Proof. By Lemma 2.1, we have that $P_{i}-P_{i+n}=a\left(U_{i}-U_{i+n}\right)$, for some constant $a$. Since

$$
w(P)\left(V_{i+\frac{1}{2}}\right)=h(P)\left(V_{i+\frac{1}{2}}\right)+h(P)\left(-V_{i+\frac{1}{2}}\right)=\left[P_{i}-P_{i+n}, V_{i+\frac{1}{2}}\right],
$$

we obtain

$$
w(P)\left(V_{i+\frac{1}{2}}\right)=2 a,
$$

$1 \leq i \leq 2 n$, thus proving the lemma.

Our next corollary says that in fact $U$ is homothetic to the Minkowski sum $P+(-P)$ (see [21], Th. 4.2.3).

Corollary 2.5. Let $P$ be a convex planar polygon and let $U$ be as in Lemma 2.1. Then $U$ is homothetic to $P+(-P)$.

Proof. We have that $2 a=h(P)+h(-P)=h(P+(-P))=h(2 a U)$, which implies that $P+(-P)$ is homothetic to $U$.

Corollary 2.6. Consider a centered polygon $U$ and a polygon $P$ whose sides are parallel to the corresponding sides of $U$. The following statements are equivalent:

1. P has constant $U$-width.

2. $P+(-P)$ is homothetic to $U$.

3. The corresponding diagonals of $U$ and $P$ are parallel to each other.

4. $P_{i}-P_{i+n}=2 a\left(U_{i}-U_{i+n}\right), 1 \leq i \leq n$, for some constant a.

\section{Geometric properties of polygons of constant Minkowskian width}

Consider a convex polygon $P=\left\{P_{1}, \ldots, P_{2 n}\right\}$ with parallel opposite sides and let $U=$ $\left\{U_{1}, \ldots, U_{2 n}\right\}$ be the symmetric polygon obtained from $P$ by the construction of Lemma 2.1 .

\subsection{Central Equidistant, $V$-length, and Barbier's theorem}

Central equidistant Any equidistant can be written as $P_{i}(c)=P_{i}+c U_{i}, 1 \leq i \leq 2 n$. If we take $c=-a$, we obtain

$$
M_{i}=P_{i}+\frac{c}{2 a}\left(P_{i}-P_{i+n}\right)=\frac{1}{2}\left(P_{i}+P_{i+n}\right), 1 \leq i \leq 2 n,
$$

called the central equidistant of $P$. It is characterized by the condition $M_{i}=M_{i+n}$ (see Figure 5). If we re-scale the one-parameter family of equidistants as

$$
P_{i}(c)=M_{i}+c U_{i}, 1 \leq i \leq 2 n,
$$


we get that the 0 -equidistant is exactly the central equidistant.

A vertex $M_{i}$ of the central equidistant is called a cusp if $M_{i-1}$ and $M_{i+1}$ are in the same half-plane defined by the diagonal at $P_{i}$. The central equidistant coincides with the area evolute of polygons defined in [5]. There it is proved that it has an odd number of cusps, at least three (see Figures 5 and 7).

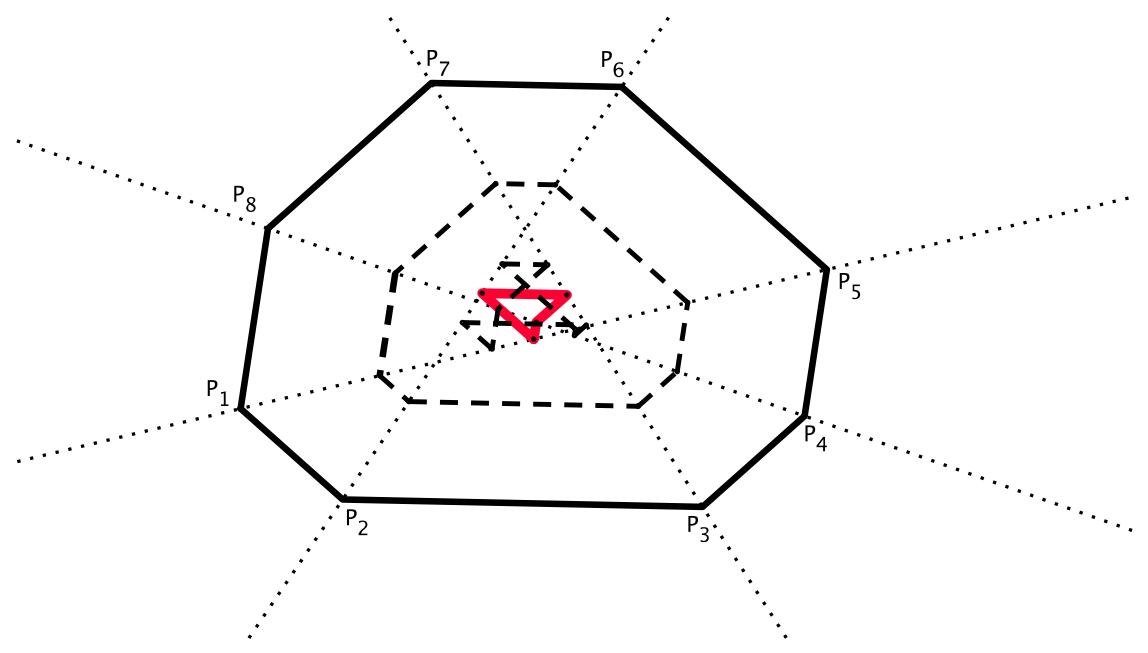

Figure 5: The two traced octagons are ordinary equidistants. The thick quadrangle is the central equidistant.

$V$-Length Let $P$ be a polygonal arc whose sides are parallel to the corresponding ones of $U$. More precisely, we shall denote by $\left\{P_{s}, \ldots, P_{t}\right\}$ the vertices of $P$ and assume that $P_{i+1}-P_{i}$ is parallel to $V_{i+\frac{1}{2}}$. We can write

$$
P_{i+1}-P_{i}=\lambda_{i+\frac{1}{2}} V_{i+\frac{1}{2}}
$$

for some $\lambda_{i+\frac{1}{2}} \geq 0$. Then the $V$-length of the edge $P_{i} P_{i+1}$ is exactly $\lambda_{i+\frac{1}{2}}$, and we write

$$
L_{V}(P)=\sum_{i=s}^{t-1} \lambda_{i+\frac{1}{2}} .
$$

Barbier's theorem The classical Theorem of Barbier on curves of constant width in the Euclidean plane says that any such curve of diameter $d$ has circumference $d \pi$. For Minkowski planes, it appears in [16], Th. 6.14(a), and in [12]. We prove here the version of this theorem for polygons.

Define $\alpha_{i+\frac{1}{2}}, 1 \leq i \leq 2 n$, by the equation

$$
M_{i+1}-M_{i}=\alpha_{i+\frac{1}{2}}\left(U_{i+1}-U_{i}\right)=\alpha_{i+\frac{1}{2}}\left[U_{i}, U_{i+1}\right] V_{i+\frac{1}{2}}
$$

Proposition 3.1. Let $P(c)$ be defined by equation (3.2). Then the $V$-length of $P(c)$ is

$$
L_{V}(P)=2 c A(U),
$$

where $A(U)$ denotes the area of the polygon $U$. 
Proof. The $V$-length of the polygon $P(c)$ is given by

$$
L_{V}(P(c))=\sum_{i=1}^{2 n}\left(\alpha_{i+\frac{1}{2}}+c\right)\left[U_{i}, U_{i+1}\right] .
$$

Since $\alpha_{i+n+\frac{1}{2}}=-\alpha_{i+\frac{1}{2}}$, we obtain

$$
L_{V}(P(c))=c \sum_{i=1}^{2 n}\left[U_{i}, U_{i+1}\right],
$$

which proves the proposition.

If we admit signed lengths, equation (3.6) holds even for equidistants with cusps. In particular, for $c=0$ we obtain

$$
L_{V}(M)=0 .
$$

For smooth closed curves this result was obtained in [19] .

\subsection{Curvature and evolutes}

Minkowskian normals and evolutes In the smooth case, the Minkowskian normal at a point $P$ is the line $P+s U$, where $P$ and $U$ have parallel tangents (see [19]). The evolute is the envelope of Minkowskian normals. For a polygon $P$, define the Minkowskian normal at a vertex $P_{i}$ as the line $P_{i}+s U_{i}, 1 \leq i \leq 2 n$, and the evolute as the polygonal arc whose vertices are the intersections of $P_{i}+s U_{i}$ and $P_{i+1}+s U_{i+1}$. These intersections are given by

$$
E_{i+\frac{1}{2}}=P_{i}-\mu_{i+\frac{1}{2}} U_{i}=P_{i+1}-\mu_{i+\frac{1}{2}} U_{i+1},
$$

where $\mu_{i+\frac{1}{2}}, 1 \leq i \leq 2 n$, is defined by

$$
P_{i+1}-P_{i}=\mu_{i+\frac{1}{2}}\left(U_{i+1}-U_{i}\right)
$$

Curvature center and radius In [16], three different notions of Minkowskian curvature are defined, where the circular curvature is directly related to evolutes. The circular center $E$ and the corresponding radius of curvature $\mu$ are defined by the condition that $E+\mu U$ has a 3-order contact with the curve at a given point (see [19]).

For polygons, we define the center of curvature $E_{i+\frac{1}{2}}$ and the curvature radius $\mu_{i+\frac{1}{2}}$ of the side $P_{i} P_{i+1}$ by the condition that the $\left(i+\frac{1}{2}\right)$-side of $E_{i+\frac{1}{2}}+\mu_{i+\frac{1}{2}} U$ matches exactly $P_{i} P_{i+1}$ (see Figure 6). Thus we get equations (3.8) and (3.9). From equations (3.3) and (3.9) we obtain that the curvature radius of the side $P_{i} P_{i+1}$ is also given by

$$
\mu_{i+\frac{1}{2}}=\frac{\lambda_{i+\frac{1}{2}}}{\left[U_{i}, U_{i+1}\right]} .
$$

A vertex $E_{i+\frac{1}{2}}$ is a cusp of the evolute if the vertices $E_{i-\frac{1}{2}}$ and $E_{i+\frac{3}{2}}$ are in the same half-plane defined by the parallel to $P_{i} P_{i+1}$ through $E_{i+\frac{1}{2}}$. The evolute of a CW polygon coincides with its center symmetry set as defined in [5], where it is proved that it coincides with the union of cusps of all equidistants of $P$. It is also proved in [5] that the number of cusps of the evolute is odd and at least the number of cusps of the central equidistant (see Figure 7). 


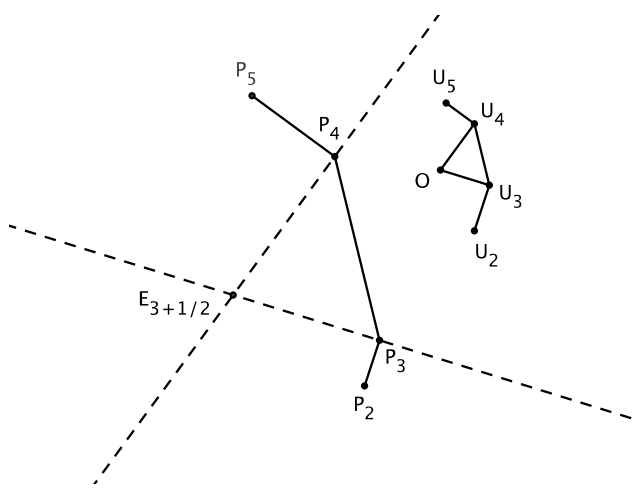

Figure 6: The center of curvature of the side $P_{3} P_{4}$.

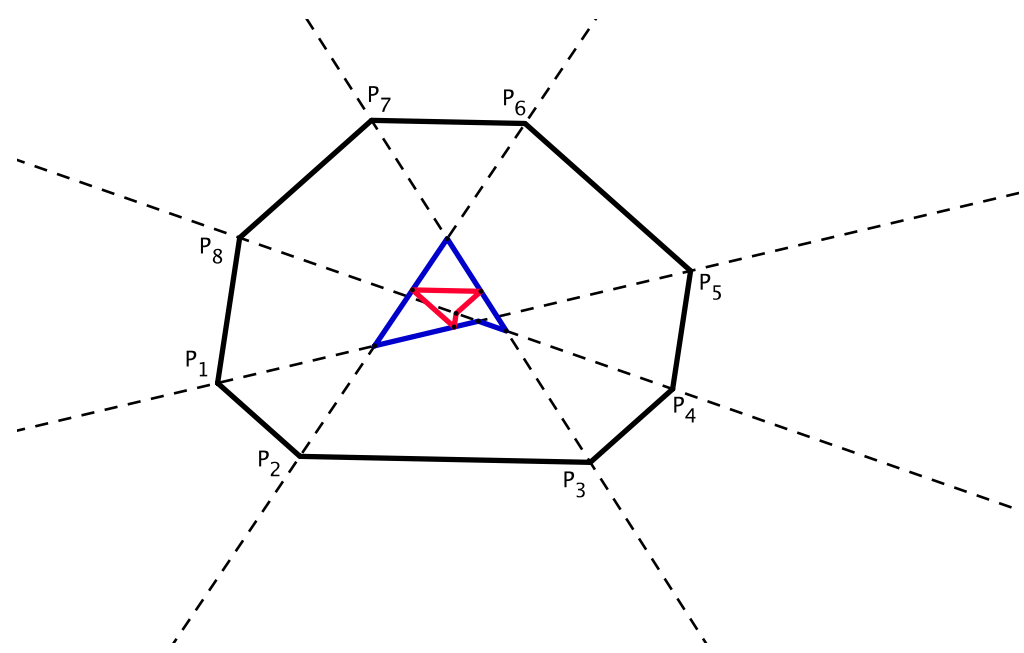

Figure 7: The inner polygonal arc is the central equidistant $M$ of $P$, and the outer polygonal arc is its evolute $E$.

Sum of curvature radii Consider equation (3.9) for two opposite sides, and sum up to obtain, for $1 \leq i \leq n$,

$$
P_{i+1}-P_{i+n+1}+P_{i+n}-P_{i}=\left(\mu_{i+\frac{1}{2}}+\mu_{i+n+\frac{1}{2}}\right)\left(U_{i+1}-U_{i}\right) .
$$

Since $P$ has constant Minkowskian width,

$$
2 c\left(U_{i+1}-U_{i}\right)=\left(\mu_{i+\frac{1}{2}}+\mu_{i+n+\frac{1}{2}}\right)\left(U_{i+1}-U_{i}\right) .
$$

We conclude that

$$
\mu_{i+\frac{1}{2}}+\mu_{i+n+\frac{1}{2}}=2 c .
$$

The corresponding result for smooth curves is given in [16], Th. 6.14.(c).

Involutes and equidistants Consider the one-parameter family of equidistants given by equation (3.2). The radius of curvature of $P_{i}(c) P_{i+1}(c)$ is the radius of curvature of 
$M_{i} M_{i+1}$ plus $c$. Thus, for $1 \leq i \leq 2 n$,

$$
E_{i+\frac{1}{2}}(c)=M_{i}+c U_{i}-\left(\mu_{i+\frac{1}{2}}+c\right) U_{i}=E_{i+\frac{1}{2}} .
$$

We conclude that the evolute of any equidistant of $P$ is equal to the evolute of $P$. Reciprocally, any polygonal arc whose evolute is equal to $E(P)$ is an equidistant of $P$. We define an involute of $E$ as any polygonal arc whose evolute is $E$. Thus the involutes of $E$ are the equidistants of $P$.

\subsection{The signed area of the central equidistant}

For a simple closed curve $P$, denote by $A(P)$ the area of the region bounded by $P$. Given two closed curves $P$ and $Q$, their mixed area is defined by the equation

$$
A(P+t Q)=A(P)+2 t A(P, Q)+t^{2} A(Q),
$$

(see $[17, \S \S 5.1]$ ). The Minkowski inequality says that $A(P, Q)^{2} \geq A(P) A(Q)$. The next lemma is well-known, see [10, $\S \S 6.3]$.

Lemma 3.2. Take $P$ and $Q$ as convex polygons with $k$ parallel corresponding sides. The mixed area of $P$ and $Q$ is given by

$$
A(P, Q)=\frac{1}{2} \sum_{i=1}^{k}\left[Q_{i}, P_{i+1}-P_{i}\right]=\frac{1}{2} \sum_{i=1}^{k}\left[P_{i+1}, Q_{i+1}-Q_{i}\right] .
$$

Assume that $P$ is a closed convex polygon whose sides are parallel to the sides of the centered polygon $U$, and take $Q=U$ in Lemma 3.2. We obtain

$$
A(P, U)=\frac{1}{2} \sum_{i=1}^{2 n}\left[U_{i}, P_{i+1}-P_{i}\right]=\frac{1}{2} \sum_{i=1}^{2 n} \lambda_{i+\frac{1}{2}}=\frac{1}{2} L_{V}(P),
$$

where we have used (3.3) and (3.4). Moreover, the Minkowski inequality becomes

$$
L_{V}^{2}(P) \geq 4 A(U) A(P) .
$$

Lemma 3.3. Let $M$ be the central equidistant of a $C W$-polygon $P$. Then the mixed area $A(M, M)$ is non-positive.

Proof. Let $P(c)$ be defined by equation (3.2). Then

$$
A(P(c), P(c))=A(M, M)+2 c A(M, U)+c^{2} A(U, U) .
$$

Now equation (3.7) says that $A(M, U)=0$. Moreover, the isoperimetric inequality (3.13) for curves of constant width says that

$$
A(P) \leq c^{2} A(U)
$$

We conclude that

$$
A(M, M) \leq 0
$$

Define the signed area of $M$ as $S A(M)=-A(M, M)$. In general, the signed area is a sum of positive and negative areas, but when $M$ is a simple curve, it coincides with the area bounded by $M$. 


\subsection{Relation between length and area of a half polygon}

Define $\beta_{i}$ by

$$
\beta_{i}=\frac{1}{2} \sum_{j=i}^{n+i-1} \alpha_{j+\frac{1}{2}}\left[U_{j}, U_{j+1}\right] .
$$

Observe that $\beta_{i+n}=-\beta_{i}, 1 \leq i \leq n$, and

$$
\beta_{i+1}-\beta_{i}=-\alpha_{i+\frac{1}{2}}\left[U_{i}, U_{i+1}\right]
$$

Denote by $A_{1}(i, c)$ and $A_{2}(i, c)$ the areas of the polygons with vertices $\left\{P_{i}, P_{i+1}, \ldots, P_{i+n}\right\}$ and $\left\{P_{i+n}, P_{i+n+1}, \ldots, P_{i}\right\}$. Observe that these polygons are bounded by $P$ and the diagonal $P_{i} P_{i+n}$.

Proposition 3.4. We have that

$$
A_{1}(i, c)-A_{2}(i, c)=4 c \beta_{i}
$$

for $1 \leq i \leq 2 n$.

Proof. Lemma 4.1. of [5] says that

$$
\begin{gathered}
A_{1}(i, c)-A_{2}(i, c)=-2 \sum_{j=i}^{i+n-1}\left[M_{j+1}-M_{j}, c U_{j}\right] \\
=-2 c \sum_{j=i}^{i+n-1}\left[\alpha_{j+\frac{1}{2}}\left[U_{j}, U_{j+1}\right] V_{j+\frac{1}{2}}, U_{j}\right] .
\end{gathered}
$$

Thus

$$
A_{1}(i, c)-A_{2}(i, c)=2 c \sum_{j=i}^{i+n-1} \alpha_{j+\frac{1}{2}}\left[U_{j}, U_{j+1}\right]=4 c \beta_{i} .
$$

Denote by $L_{V}(i, c)$ the $V$-length of the polygonal arc whose vertices are $\left\{P_{i}(c), P_{i+1}(c), \ldots\right.$, $\left.P_{i+n}(c)\right\}$. Then

$$
L_{V}(i, c)=\sum_{j=i}^{i+n-1}\left(\alpha_{i+\frac{1}{2}}+c\right)\left[U_{j}, U_{j+1}\right]=2 c A(U)+2 \beta_{i} .
$$

Corollary 3.5. For $1 \leq i \leq 2 n$, the expression $A_{1}(i, c)-c L_{V}(i, c)$ is independent of $i$.

Proof. By equation (3.16) and Proposition 3.4, we get

$$
2 c L_{V}(i, c)-2 A_{1}(i, c)=4 c^{2} A(U)+4 c \beta_{i}-2 A_{1}(i, c)=4 c^{2} A(U)-A(P),
$$

which proves the corollary.

The above corollary presents the "polygonal analogue" of a known theorem holding for strictly convex curves (see [4], eq. (2.1)). 


\section{The involute of the central equidistant}

Recall that $P=\left\{P_{1}, \ldots, P_{2 n}\right\}$ is a convex polygon with parallel opposite sides and $U=$ $\left\{U_{1}, \ldots, U_{2 n}\right\}$ is the Minkowski ball obtained from $P$ by the construction of Lemma 2.1. The polygon $V=\left\{V_{1}, \ldots, V_{2 n}\right\}$ represents the dual Minkowski ball (see Lemma 2.3) and $M=\left\{M_{1}, \ldots, M_{n}\right\}$ is the central equidistant of $P$ (see equation (3.1)).

\subsection{Basic properties of the involute $N$ of $M$}

Define the polygon $N$ by

$$
N_{i+\frac{1}{2}}=M_{i}+\beta_{i} V_{i+\frac{1}{2}}
$$

$1 \leq i \leq 2 n$. Observe that $N_{i+\frac{1}{2}}=N_{i+n+\frac{1}{2}}$. Due to equations (3.5) and (3.15), we can also write

$$
N_{i+\frac{1}{2}}=M_{i+1}+\beta_{i+1} V_{i+\frac{1}{2}} .
$$

Lemma 4.1. The polygon $N$ has constant $V$-width, and the evolute of $N$ is $M$.

Proof. Since

$$
N_{i+\frac{1}{2}}-N_{i-\frac{1}{2}}=\beta_{i}\left(V_{i+\frac{1}{2}}-V_{i-\frac{1}{2}}\right),
$$

$1 \leq i \leq n$, the sides of $N$ are parallel to the sides of $V$. Moreover, the diagonals of $N$ are zero, so they are multiples of the diagonals of $V$. We conclude from Corollary 2.6 that $N$ has constant $V$-width. Finally, from equation (4.1) we conclude that the evolute of $N$ is $M$.

The equidistants of $N$, which are the involutes of $M$, are curves of constant $V$-width (see Figure 8). In [5], these polygons were called the Parallel Diagonal Transforms of $P$.

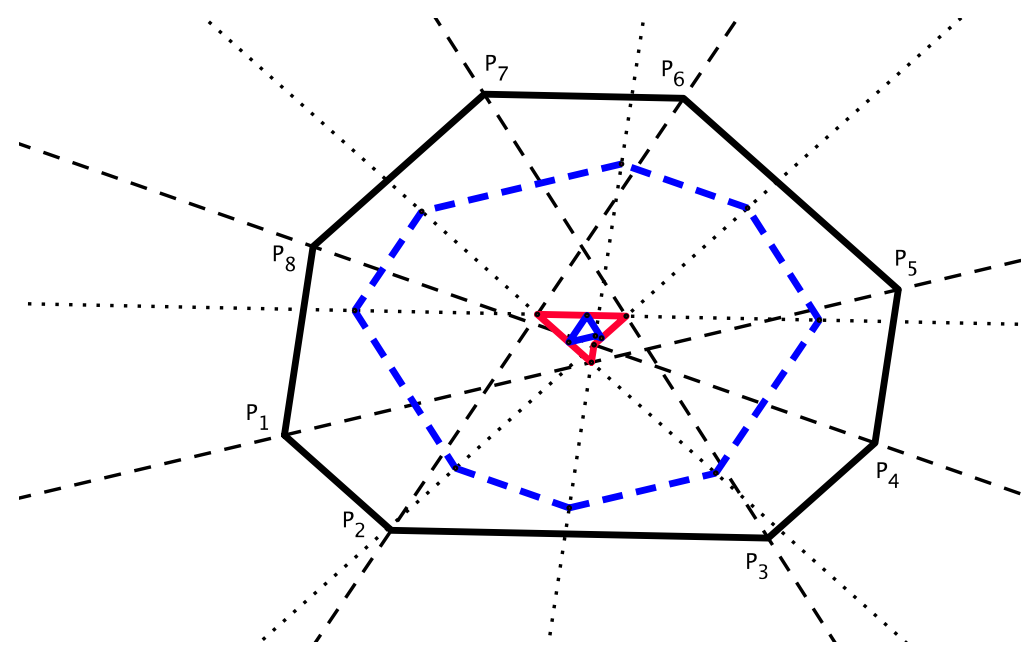

Figure 8: The central equidistant $M$ together with two involutes of $M$ : The inner curve is the central equidistant $N$, and the traced curve is an ordinary involute. 


\subsection{The signed area of the involute of the central equidistant}

For smooth convex curves of constant Minkowskian width, the signed area of $N$ is not larger than the signed area of $M$ (see [6]). We prove here the corresponding result for polygons.

Proposition 4.2. Denoting by $S A(M)$ and $S A(N)$ the signed areas of $M$ and $N$, we have

$$
S A(M)-S A(N)=\sum_{i=1}^{n} \beta_{i}^{2}\left[V_{i-\frac{1}{2}}, V_{i+\frac{1}{2}}\right] .
$$

Proof. Observe that

$$
\begin{gathered}
{\left[M_{i}, M_{i+1}\right]=\left[N_{i+\frac{1}{2}}-\beta_{i} V_{i+\frac{1}{2}}, \alpha_{i+\frac{1}{2}}\left(U_{i+1}-U_{i}\right)\right]=\alpha_{i+\frac{1}{2}}\left[N_{i+\frac{1}{2}}, U_{i+1}-U_{i}\right]=} \\
-\left(\beta_{i+1}-\beta_{i}\right)\left[N_{i+\frac{1}{2}}, V_{i+\frac{1}{2}}\right], \quad\left[N_{i-\frac{1}{2}}, N_{i+\frac{1}{2}}\right]=\beta_{i}\left[N_{i+\frac{1}{2}}, V_{i+\frac{1}{2}}-V_{i-\frac{1}{2}}\right],
\end{gathered}
$$

and so

$$
-\left[M_{i}, M_{i+1}\right]+\left[N_{i-\frac{1}{2}}, N_{i+\frac{1}{2}}\right]=\left[N_{i+\frac{1}{2}}, \beta_{i+1} V_{i+\frac{1}{2}}-\beta_{i} V_{i-\frac{1}{2}}\right] .
$$

Thus

$$
\begin{aligned}
& S A(M)-S A(N)=\sum_{i=1}^{n}-\left[M_{i}, M_{i+1}\right]+\left[N_{i-\frac{1}{2}}, N_{i+\frac{1}{2}}\right]= \\
& =-\sum_{i=1}^{n}\left[N_{i+\frac{1}{2}}-N_{i-\frac{1}{2}}, \beta_{i} V_{i-\frac{1}{2}}\right]=\sum_{i=1}^{n} \beta_{i}^{2}\left[V_{i-\frac{1}{2}}, V_{i+\frac{1}{2}}\right]
\end{aligned}
$$

where we have used that the difference

$$
\left[N_{i+\frac{1}{2}}, \beta_{i+1} V_{i+\frac{1}{2}}\right]-\left[N_{i-\frac{1}{2}}, \beta_{i} V_{i-\frac{1}{2}}\right]
$$

is equal to

$$
\left[N_{i+\frac{1}{2}}-N_{i-\frac{1}{2}}, \beta_{i} V_{i-\frac{1}{2}}\right]+\left[N_{i+\frac{1}{2}}, \beta_{i+1} V_{i+\frac{1}{2}}-\beta_{i} V_{i-\frac{1}{2}}\right],
$$

the discrete version of "integration by parts".

\subsection{The involute is contained in the interior of the central equidistant}

We prove now that the region bounded by the central equidistant $M$ contains its involute $N$. For smooth convex curves, this result was proved in [6].

The exterior of the curve $M$ is defined as the set of points of the plane that can be reached from a point of $P$ by a path that does not cross $M$. The region $\bar{M}$ bounded by $M$ is the complement of its exterior. It is well known that a point in the exterior of $M$ is the center of exactly one chord of $P$ (see [5]).

Proposition 4.3. The involute $N$ is contained in the region $\bar{M}$ bounded by $M$.

The proof is based on two lemmas. For a fixed index $i$, denote by $l(i)$ the line parallel to $P_{i+n}-P_{i}$ through $N_{i-\frac{1}{2}}$ and $N_{i+\frac{1}{2}}$. Then $l(i)$ divides the interior of $P$ into two regions of areas $B_{1}=B_{1}(i)$ and $B_{2}=B_{2}(i)$, where the second one contains $P_{i}$ and $P_{i+n}$. 
Lemma 4.4. We have that $B_{1}(i) \geq B_{2}(i), 1 \leq i \leq n$.

Proof. We have that

$$
B_{1}(i)=A_{1}(i)-\left(2 c \beta_{i}-\delta_{i}-\eta_{i}\right), \quad B_{2}(i)=A_{2}(i)+\left(2 c \beta_{i}-\delta_{i}-\eta_{i}\right),
$$

where $\delta_{i}$ is the area of the regions outside $P$ and between $l(i), P_{i} P_{i+n}$ and the support lines of $P_{i} P_{i+1}$ and $P_{i+n-1} P_{i+n}$, and $\eta_{i}$ is the area of the triangle $M_{i} N_{i+\frac{1}{2}} N_{i-\frac{1}{2}}$ (see Figure 9). Since, by Proposition $3.4,4 c \beta_{i}=A_{1}-A_{2}$, we conclude that

$$
B_{1}(i)=\frac{A(P)}{2}+\delta_{i}+\eta_{i}, \quad B_{2}(i)=\frac{A(P)}{2}-\delta_{i}-\eta_{i},
$$

which proves the lemma.

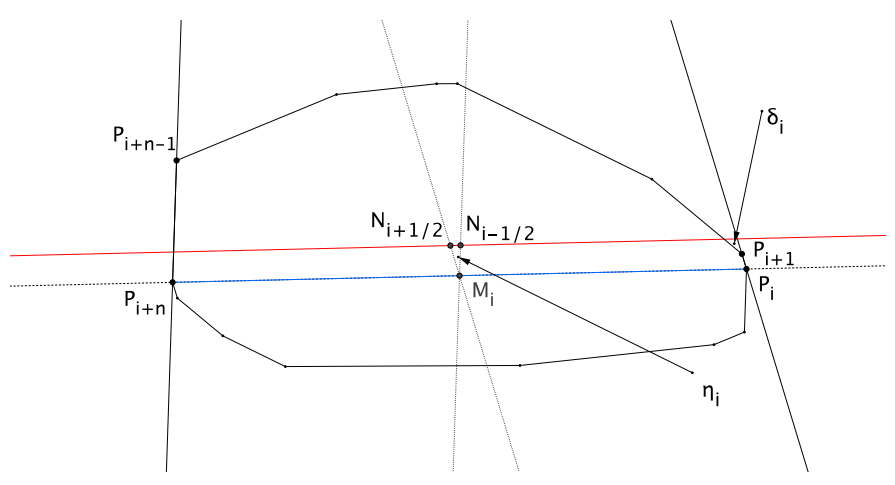

Figure 9: The line through $N_{i+\frac{1}{2}}$ and $N_{i-\frac{1}{2}}$ divides the polygon into two regions of areas $B_{1}$ and $B_{2}$.

Lemma 4.5. Choose $C$ in the segment $N_{i-\frac{1}{2}} N_{i+\frac{1}{2}}$. Then $C$ is in the region bounded by $M$.

Proof. By an affine transformation of the plane, we may assume that $l(i)$ and $M_{i} C$ are orthogonal. Consider polar coordinates $(r, \phi)$ with center $C$ and describe $P$ by $r(\phi)$. Assume that $\phi=0$ at the line $l(i)$ and that $\phi=-\phi_{0}$ at $P_{i}$. Denote the area of the sector bounded by $P$ and the rays $\phi_{1}, \phi_{2}$ by

$$
A\left(\phi_{1}, \phi_{2}\right)=\frac{1}{2} \int_{\phi_{1}}^{\phi_{2}} r^{2}(\phi) d \phi .
$$

Consider a line parallel to $M_{i} C$ and passing through the point $Q_{0}$ of $P$ corresponding to $\phi=0$, and denote by $Q_{1}$ and $Q_{2}$ its intersection with the rays $\phi=-\phi_{0}$ and $\phi=\phi_{0}$, respectively (see Figure 10). By convexity, we have that

$$
A\left(0, \phi_{0}\right) \leq A\left(C Q_{0} Q_{1}\right)=A\left(C Q_{0} Q_{2}\right) \leq A\left(-\phi_{0}, 0\right) .
$$

A similar reasoning shows that $A\left(\pi-\phi_{0}, \pi\right) \leq A\left(\pi, \pi+\phi_{0}\right)$. Observe also that, by convexity, $r\left(\phi_{0}\right) \leq r\left(\phi_{0}+\pi\right)$ and $r\left(\pi-\phi_{0}\right) \leq r\left(-\phi_{0}\right)$. 
Now, if $r(\phi+\pi)>r(\phi)$ for any $\phi_{0}<\phi<\pi-\phi_{0}$, we would have $B_{1}(C)<B_{2}(C)$, contradicting the previous lemma. We conclude that $r(\phi+\pi)=r(\phi)$ for at least two values of $\phi_{0}<\phi<\pi-\phi_{0}$. Since equality holds also for some $\pi-\phi_{0}<\phi<\pi+\phi_{0}$, there are at least three chords of $\gamma$ having $C$ as midpoint. Thus $C$ is contained in the region bounded by $M$.

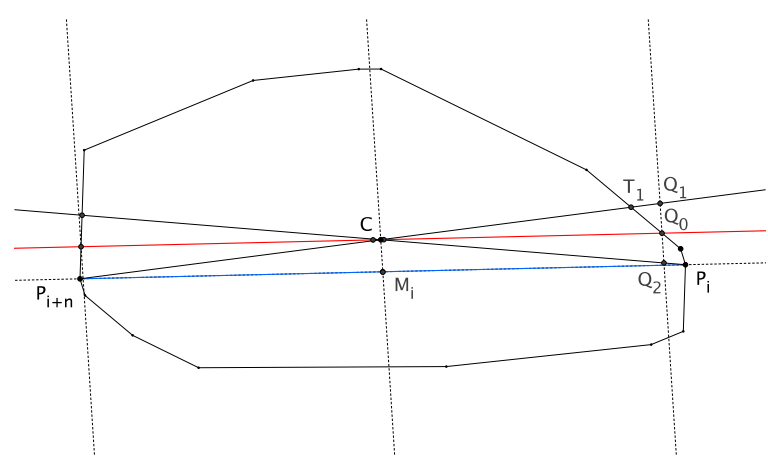

Figure 10: The line parallel to $M_{i} C$ through $Q_{0}$ determines the points $Q_{1}$ and $Q_{2}$.

We can now complete the proof of Proposition 4.3. In fact, from Lemma 4.5 we have that each side $N_{i-\frac{1}{2}} N_{i+\frac{1}{2}}$ is contained in the region $\bar{M}$ bounded by $M$. Therefore, no point on the boundary of $N$ can be connected with the boundary of $P$ by a curve that does not intersect $M$. This implies that the region $\bar{N}$ bounded by $N$ is contained in $\bar{M}$.

\section{Iterating involutes}

Starting with the central equidistant $M=M(0)$ and its involute $N=N(1)$, we can iterate the involute operation. We obtain two sequences of $n$-gons $M(k)$ and $N(k)$ defined by $M(k)=\mathcal{I} n v(N(k))$ and $N(k+1)=\mathcal{I} n v(M(k))$. For smooth curves of constant Minkowskian width, it is proved in [6] that these sequences converge to a constant. We prove here the corresponding result for polygons.

From Proposition 4.3, we have

$$
\overline{M(0)} \supset \overline{N(1)} \supset \overline{M(1)} \supset \ldots,
$$

and we denote by $O=O(P)$ the intersection of all these sets.

If we represent a polygon by its vertices, we can embed the space $\mathcal{P}_{n}$ of all $n$-gons in $\left(\mathbb{R}^{2}\right)^{n}$. In $\mathcal{P}_{n}$ we consider the topology induced by $\mathbb{R}^{2 n}$.

Theorem 5.1. The set $O=O(P)$ consists of a unique point, and the polygons $M(k)$ and $N(k)$ are converging to $O$ in $\mathcal{P}_{n}$.

We shall call $O=O(P)$ the central point of $P$. A natural question that arises is the following.

Question Is there a direct method to obtain the central point $O$ from the polygon $P$ ? 
For fixed $c$ and $d$ construct the sequences of convex polygons $P(k, c)$ and $Q(k, d)$ whose vertices are

$$
P_{i}(k)=M_{i}(k)+c U_{i}(k), Q_{i+\frac{1}{2}}(k)=N_{i+\frac{1}{2}}(k)+d V_{i+\frac{1}{2}}(k),
$$

respectively. The polygons $P(k, c)$ are of constant $U$-width, while the polygons $Q_{i+\frac{1}{2}}(k, d)$ are of constant $V$-width. We can re-state Theorem 5.1 as follows:

Theorem 5.2. The sequences of polygons $P(k, c)$ and $Q(k, d)$ are converging in $\mathcal{P}_{2 n}$ to $O+c \partial \mathcal{U}$ and $O+d \partial \mathcal{V}$, respectively.

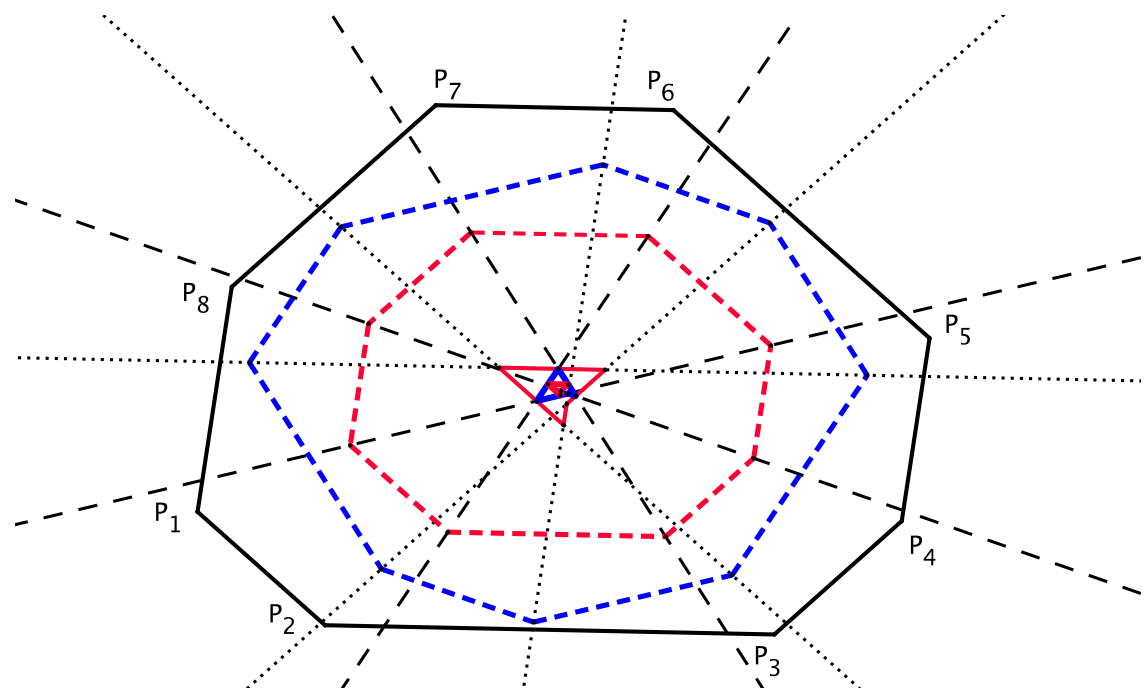

Figure 11: The inner curves are $M=M(0), N=N(1)$ and $M(1)$. One traced curve is an ordinary $V$-equidistant of $N$, and the other one is an ordinary $U$-equidistant of $M(1)$.

We shall prove now Theorem 5.1.

Proof. Denote the signed areas of $M(k)$ and $N(k)$ by $S A(M(k))$ and $S A(N(k))$, respectively. By Section 3.3, $S A(M(k)) \geq 0, S A(N(k)) \geq 0$, and Proposition 4.2 implies that

$$
\begin{gathered}
S A(M(k))-S A(N(k+1))=\sum_{i=1}^{n} \beta_{i}^{2}(k)\left[U_{i}, U_{i+1}\right], \\
S A(N(k))-S A(M(k))=\sum_{i=1}^{n} \alpha_{i+\frac{1}{2}}^{2}(k)\left[V_{i-\frac{1}{2}}, V_{i+\frac{1}{2}}\right],
\end{gathered}
$$

where $\alpha_{i+\frac{1}{2}}(k)$ and $\beta_{i}(k)$ are defined by

$$
\begin{aligned}
& M_{i+1}(k)-M_{i}(k)=\alpha_{i+\frac{1}{2}}(k)\left(U_{i+1}-U_{i}\right), \\
& N_{i+\frac{1}{2}}(k)-N_{i-\frac{1}{2}}(k)=\beta_{i}(k)\left(V_{i+\frac{1}{2}}-V_{i-\frac{1}{2}}\right) .
\end{aligned}
$$


We conclude that

$$
\sum_{k=1}^{\infty} \sum_{i=1}^{n} \beta_{i}^{2}(k)\left[U_{i}, U_{i+1}\right]+\sum_{k=0}^{\infty} \sum_{i=1}^{n} \alpha_{i+\frac{1}{2}}^{2}(k)\left[V_{i-\frac{1}{2}}, V_{i+\frac{1}{2}}\right] \leq S A(M(0)) .
$$

From the above equation, we obtain that the sequences $\alpha_{i+\frac{1}{2}}(k)$ and $\beta_{i}(k)$ are converging to 0 in $\mathbb{R}^{n}$. So the diameters of $M(k)$ and $N(k)$ are converging to zero, and thus $O$ is in fact a set consisting of a unique point.

\section{References}

[1] R. Ait-Haddou, L. Biard and M.A. Slawinski, Minkowski isoperimetric-hodograph curves, Computer Aided Geometric Design 17 (2000), 835-861.

[2] T.M. Apostol and M.A. Mnatsakanian, Tanvolutes: generalized involutes, Amer. Math. Monthly 117 (2010), 701-713.

[3] G.D. Chakerian, Sets of constant width, Pacific J. Math. 19(1) (1966), 13-21.

[4] G.D. Chakerian and H. Groemer, Convex bodies of constant width, in: P.M. Gruber and J.M. Wills (eds.), Convexity and its Applications, Birkhäuser, Basel, 1983, 49-96.

[5] M. Craizer, R.C. Teixeira and M.A.H.B da Silva, Polygons with parallel opposite sides, Discrete and Computational Geometry 50(2) (2013), 474-490.

[6] M. Craizer, Iteration of involutes of constant width curves in the Minkowski plane, Beitr. Algebra Geom. 55 (2014), 479-496.

[7] P.J. Giblin, Affinely invariant symmetry sets, Geometry and Topology of Caustics (Caustics 06), Banach Center Publications 82 (2008), 71-84.

[8] A. Gray, Modern Differential Geometry of Curves and Surfaces, Studies in Advanced Mathematics, CRC Press, Boca Raton, 1993.

[9] A. Gray, E. Abbena and S. Salamon, Modern Differential Geometry of Curves and Surfaces with Mathematica, Studies in Advanced Mathematics, Chapman \& Hall/CRC, Boca Raton, 2006.

[10] P.M. Gruber, Convex and Discrete Geometry, Springer, Berlin and Heidelberg, 2007.

[11] E. Heil and H. Martini, Special convex bodies, in: P.M. Gruber, J.M. Wills (eds.), Handbook for Convex Geometry, North-Holland, Amsterdam, 1993, 347-385.

[12] H. Martini and Z. Mustafaev, On Reuleaux triangles in Minkowski planes, Beitr. Algebra Geom. 48 (2007), 225-235.

[13] H. Martini and K.J. Swanepoel, The geometry of Minkowski spaces - a survey. Part II, Expositiones Math. 22 (2004), 93-144.

[14] H. Martini, K.J. Swanepoel and G. Weiss, The geometry of Minkowski spaces - a survey. Part I, Expositiones Math. 19 (2001), 97-142.

[15] H. Martini and Senlin Wu, Classical curve theory in normed planes, Computer Aided Geometric Design 31 (2014), 373-397.

[16] C.M. Petty, On the geometry of the Minkowski plane, Riv. Mat. Univ. Parma 6 (1955), 269292.

[17] R. Schneider, Convex Bodies:The Brunn-Minkowski Theory, Encyclopedia of Mathematics and its Applications, 44, Cambridge University Press, (1993).

[18] P.A. Solov'ev: Maximum length of the closed involute of a class of curves (Russian), Ukrain. Geom. Sb. 3 (1966), 112-122. 
[19] S. Tabachnikov: Parameterized plane curves, Minkowski caustics, Minkowski vertices and conservative line fields, L'Enseign. Math. 43 (1997), 3-26.

[20] S. Tanno: $C^{\infty}$-approximation of continuous ovals of constant width, J. Math. Soc. Japan 28 (1976), 384-395.

[21] A.C. Thompson: Minkowski Geometry, Encyclopedia of Mathematics and its Applications, 63, Cambridge University Press, 1996.

[22] D. Yost: Irreducible convex sets, Mathematika 38 (1991), 134-155. 